PROF. MARIANO SANZ (Orcid ID : 0000-0002-6293-5755)

DR. DAVID HERRERA (Orcid ID : 0000-0002-5554-2777)

DR. MORITZ KEBSCHULL (Orcid ID : 0000-0003-1863-0679)

PROF. SØREN JEPSEN (Orcid ID : 0000-0002-4160-5837)

PROF. TORD BERGLUNDH (Orcid ID : 0000-0001-5864-6398)

PROF. ANTON SCULEAN (Orcid ID : 0000-0003-2836-5477)

Article type : Supplement Article

\title{
1. TITLE
}

Treatment of Stage I-III Periodontitis -The EFP S3 Level Clinical Practice

\section{Guideline}

\section{ii. SHORT RUNNING TITLE}

Treatment of Periodontitis Guideline

\section{iii. Full names of the authors}

Mariano Sanz ${ }^{1}$, David Herrera ${ }^{1}$, Moritz Kebschull2,3,4, Iain Chapple ${ }^{2,3}$, Søren Jepsen ${ }^{5}$, Tord Beglundh $^{6}$, Anton Sculean 7 , Maurizio S. Tonetti ${ }^{8,9}$ *

* On behalf of the EFP workshop participants and methodological consultants (listed below).

\section{iv. Author's institutional affiliations}

1. ETEP (Etiology and Therapy of Periodontal and Peri-implant Diseases) Research Group, University Complutense of Madrid, Spain.

2. Periodontal Research Group, Institute of Clinical Sciences, College of Medical \& Dental

Sciences, The University of Birmingham, Birmingham, United Kingdom.

3. Birmingham Community Healthcare NHS Trust, Birmingham, United Kingdom.

This article has been accepted for publication and undergone full peer review but has not been through the copyediting, typesetting, pagination and proofreading process, which may lead to differences between this version and the Version of Record. Please cite this article as doi: 10.1111/JCPE. 13290

This article is protected by copyright. All rights reserved 
4. Division of Periodontics, Section of Oral, Diagnostic and Rehabilitation Sciences, College of Dental Medicine, Columbia University, New York, NY, USA

5. Department of Periodontology, Operative and Preventive Dentistry. University Hospital Bonn, Bonn, Germany.

6. Department of Periodontology, Institute of Odontology, The Sahlgrenska Academy, University of Gothenburg, Göteborg, Sweden

7. Department of Periodontology, School of Dental Medicine, University of Bern, Bern, Switzerland.

8. Division of Periodontology and Implant Dentistry, Faculty of Dentistry, the University of Hong Kong, Hong Kong.

9. Department of Oral and Maxillo-facial Implantology, Shanghai Key Laboratory of Stomatology, National Clinical Research Centre for Stomatology, Shanghai Ninth People Hospital, School of Medicine, Shanghai Jiao Tong University, Shanghai, China

\section{Workshop Participants}

Anne Merete Aass, Mario Aimetti, Bahar Eren Kuru, Georgios Belibasakis, Juan Blanco, Ellen Bol-van den Hil, Nagihan Bostanci, Darko Bozic, Philippe Bouchard, Nurcan Buduneli, Francesco Cairo, Elena Calciolari, Maria Clotilde Carra, Pierpaolo Cortellini, Jan Cosyn, Francesco D’Aiuto, Bettina Dannewitz, Monique Danser, Korkud Demirel, Jan Derks, Massimo de Sanctis, Thomas Dietrich, Christof Dörfer, Henrik Dommisch, Nikos Donos, Kenneth Eaton, Peter Eickholz, Elena Figuero, William Giannobile, Moshe Goldstein, Filippo Graziani, Phophi Kamposiora, Lise-Lotte Kirkevang, Thomas Kocher, Eija Kononen, Nicklaus Lang, France Lambert, Luca Landi, Paulo Melo, Bruno Loos, Rodrigo Lopez, Pernilla Lundberg, Eli Machtei, Phoebus Madianos, Conchita Martín, Paula Matesanz, Jörg Meyle, Ana Molina, Eduardo Montero, Jose Nart, Ian Needleman, Luigi Nibali, Panos Papapanou, Andrea Pilloni, David Polak, Ioannis Polyzois, Philip Preshaw, Marc Quirynen, Christoph Ramseier, Stefan Renvert, Giovanni Salvi, Ignacio Sanz-Sánchez, Lior Shapira, Dagmar Else Slot, Andreas Stavropoulos, Xavier Struillou, Jean Suvan, Wim Teughels, Daniela Timus, Cristiano Tomasi, Leonardo Trombelli, Fridus van der Weijden, Paula Vassallo, Clemens Walter, Nicola West, Gernot Wimmer

\section{Methodological Consultants}

Ina Kopp (chief consultant), Paul Brocklehurst, Jan Wennström 


\section{Workshop Organization}

European Federation of Periodontology

\section{Scientific societies involved in the guideline development process}

European Federation of Conservative Dentistry

European Association of Dental Public Health

European Society for Endodontology

European Prosthodontic Association

\section{Other organisations involved in the guideline development process}

Council of European Dentists

European Dental Hygienists' Federation

European Dental Students' Association

Platform for Better Oral Health in Europe

\section{v. Acknowledgments}

The authors express their gratitude to all reviewers involved in the preparation of the systematic reviews. In addition, the organizations which accepted to participate in the guideline development process are also kindly and sincerely acknowledged: European Federation of Conservative Dentistry, European Association of Dental Public Health, European Society for Endodontology, European Prosthodontic Association, Council of European Dentists, European Dental Hygienists' Federation, European Dental Students' Association, Platform for Better Oral Health in Europe.

\section{Conflict of Interest Statement}

Individual potential conflict of interest forms were completed by all participants and are available on file at the European Federation of Periodontology and extracted in the Supporting Information, available on line (Final Guideline-Supporting Information_Potential conflict of interests). In addition, potential conflict of interest information of the chairs of the workshop is listed here. Dr. Sanz, Mariano (Chair) reports personal fees from Camlog implants, Colgate, Dentium Implants, Dentsply Sirona Implants, Geistlich, GSK, Klockner Implants, MIS Implants, Mozo Grau Implants, Nobel Biocare, Procter \& Gamble, Straumann, Sunstar; grants from Camlog Implants, Dentaid, Dentium Implants, Dentsply Sirona Implants, Geistlich 
Pharma, Klockner Implants, MIS Implants, Mozo Grau Implants, Nobel Biocare, Sunstar, Straumann AG, Sweden and Martina Implants; and other support from Dentaid, outside the submitted work.

Dr. Herrera, David (Chair) reports personal fees from Colgate, Dentaid, Dexcel Pharma, GSK, Johnson \& Johnson, Klockner Implants, Procter \& Gamble, Straumann; grants from Colgate, Dentaid, GSK, Kulzer, Zimmer-Biomet, outside the submitted work. Other relationships or activities reported: Trustee of the Foundation of the Spanish Society of Periodontology, board member of the Continental European Division of the International Association of Periodontology, committee member of the Posgraduate Education Committee of the European Federation of Periodontology (EFP), committee member of the European Workshop Committee of the European Federation of Periodontology (EFP), expert in the Global Periodontal Health Project (FDI World Dental Federation); Specific interest in periodontal therapy, as periodontist practising on a daily basis, and in periodontal therapy, as main interest in research.

Dr. Kebschull, Moritz (Chair) reports personal fees from Colgate, Dexcel Pharma, Geistlich Pharma, Hu-Friedy, NSK, Procter \& Gamble; non-financial support from Colgate, Dexcel Pharma, Geistlich Pharma, Hu-Friedy, NSK, Procter \& Gamble, outside the submitted work.

Dr. Chapple, Iain (Chair) reports personal fees from Procter \& Gamble; grants from GSK, Unilever, outside the submitted work. In addition, Dr. Chapple has 8 patents on saliva diagnostics issued and his wife runs Oral Health Innovations that has the license for PreViser and DEPPA risk assessment software in the UK.

Dr. Jepsen, Sören (Chair) reports personal fees from Colgate, Geistlich Pharma, Procter \& Gamble, outside the submitted work.

Dr. Berglundh, Tord (Chair) reports personal fees from Dentsply Sirona Implants, Straumann; grants from Dentsply Sirona Implants, outside the submitted work.

Dr. Sculean, Anton (Chair) reports personal fees from Botiss Biomaterials, Geistlich Pharma, Oral Reconstruction Foundation, Osteology Foundation, Straumann AG, Regedent AG, Stoma; grants from Botiss Biomaterials, Geistlich Pharma, ITI Foundation, Oral Reconstruction Foundation, Osteology Foundation, Straumann AG, Regedent AG; outside the submitted work.

Dr. Tonetti, Maurizio (Chair) reports personal fees from Geistlich Pharma AG, Procter \& Gamble, Straumann AG, Sunstar SA, Unilever; grants from Geistlich Pharma, Sunstar SA; non-financial support from Procter \& Gamble, outside the submitted work.

\section{Corresponding author}

Mariano Sanz

ETEP (Etiology and Therapy of Periodontal and Peri-implant Diseases) Research Group Faculty 


\section{vi. Abstract and keywords;}

\section{Abstract}

Background: The recently introduced 2017 World Workshop classification of periodontitis, incorporating stages and grades of disease, aims to link disease classification with approaches to prevention and treatment, as it not only describes disease severity and extent, but also the degree of complexity and an individual's risk. There is, therefore, a need for evidence-based clinical guidelines providing recommendations to treat periodontitis.

Aim: The objective of the current project was to develop a S3 Level Clinical Practice Guideline (CPG) for the treatment of stage I-III periodontitis.

Material and Methods: This S3 CPG was developed under the auspices of the European Federation of Periodontology (EFP), following the methodological guidance of the Association of Scientific Medical Societies in Germany and the Grading of Recommendations Assessment, Development and Evaluation (GRADE). The rigorous and transparent process included synthesis of relevant research in 15 specifically commissioned systematic reviews, evaluation of the quality and strength of evidence, the formulation of specific recommendations and consensus, on those recommendations, by leading experts and a broad base of stakeholders.

Results: The S3 CPG approaches the treatment of periodontitis (stages I, II and III) using a preestablished stepwise approach to therapy that, depending on the disease stage, should be incremental, each including different interventions. Consensus was achieved on recommendations covering different interventions, aimed at: i) behavioural changes, supragingival biofilm, gingival inflammation and risk factor control; ii) supra- and sub-gingival instrumentation, with and without adjunctive therapies; iii) different types of periodontal surgical interventions; and iv) the necessary supportive periodontal care to extend benefits over time.

Conclusion: This S3 guideline informs clinical practice, health systems, policymakers and, indirectly, the public, on the available and most effective modalities to treat periodontitis and to maintain a healthy dentition for a lifetime, according to the available evidence at the time of publication.

Key words: periodontitis, stage, grade, clinical guideline, periodontal therapy, health policy, oral health 


\section{vii. Clinical Relevance}

\section{Scientific rationale for the study}

Implementation of the new classification of periodontitis should be facilitate the use of the most appropriate preventive and therapeutic interventions, depending on the stage and grade of the disease. The choice of these interventions should be made following a rigorous evidence-based decision-making process.

\section{Principal findings}

This guideline has been developed using strict validated methodologies for assuring the best available evidence on the efficacy of the interventions considered and the most appropriate recommendations based on a structured consensus process, including a panel of experts and representatives from key stakeholders.

\section{Practical implications}

The application of this S3 Level Clinical Practice Guideline will allow a homogeneous and evidence-based approach to the management of stages I-III periodontitis. 


\section{Introduction}

\section{The Health Problem}

\section{Definition}

Periodontitis is characterized by progressive destruction of the tooth-supporting apparatus. Its primary features include the loss of periodontal tissue support manifest through clinical attachment loss (CAL) and radiographically assessed alveolar bone loss, presence of periodontal pocketing and gingival bleeding (Papapanou et al., 2018). If untreated it may lead to tooth loss, although it is preventable and treatable in the majority of cases.

\section{Importance}

Periodontitis is a major public health problem due to its high prevalence, and since it may lead to tooth loss and disability, it negatively affects chewing function and aesthetics, is a source of social inequality, and significantly impairs quality of life. Periodontitis accounts for a substantial proportion of edentulism and masticatory dysfunction, has a negative impact on general health and results in significant dental care costs (Tonetti, Jepsen, Jin, \& Otomo-Corgel, 2017).

\section{Pathophysiology}

Periodontitis is a chronic multifactorial inflammatory disease associated with dysbiotic dental plaque biofilms.

\section{Prevalence}

Periodontitis is the most common chronic inflammatory non-communicable disease of humans. According to the Global Burden of Disease 2010 study, the global age-standardized prevalence (1990-2010) of severe periodontitis was 11.2\%, representing the sixth-most prevalent condition in the world (Kassebaum et al., 2014), while in the Global Burden of Disease 2015 study, the prevalence of severe periodontitis was estimated in 7.4\% (Kassebaum et al., 2017). The prevalence of milder forms of periodontitis may be as high as 50\% (Billings et al., 2018).

\section{Billings M, Holtfreter B, Papapanou}




\title{
PN, Mitnik GL, Kocher T, Dye BA.
}

\section{Age-dependent distribution}

\section{of periodontitis in two countries: Findings}

\author{
from NHANES
}

2009 to 2014 and SHIP-TREND 2008 to 2012.

Journal of

Clinical Periodontology. 2018; 45(Suppl

20):S130-S148.

Consequences of failure to treat

Untreated or inadequately treated periodontitis leads to the loss of tooth-supporting tissues and teeth. Severe periodontitis, along with dental caries is responsible for more years lost to disability than any other human disease (GBD 2017 Disease and Injury Incidence and Prevalence Collaborators, 2018). Furthermore, periodontal infections are associated with a range of systemic diseases leading to premature death, including diabetes (Sanz et al., 2018), cardiovascular diseases (Sanz et al., 2019; Tonetti, Van Dyke, \& Working group 1 of the joint EFP/AAP Workshop, 2013) or adverse pregnancy outcomes (Sanz, Kornman, \& Working group 3 of joint EFP/AAP Workshop, 2013).

\section{Economic importance}

On a global scale, periodontitis is estimated to cost $\$ 54$ billion in direct treatment costs and further \$25 billion in indirect costs (GBD 2017 Disease and Injury Incidence and Prevalence Collaborators, 2018). Periodontitis contributes significantly to the cost of dental diseases due to the need to replace teeth lost to periodontitis. The total cost of dental diseases, in 2015, was 
estimated to be of $\$ 544.41$ billion, being $\$ 356.80$ billion direct costs, and $\$ 187.61$ billion indirect costs (Righolt, Jevdjevic, Marcenes, \& Listl, 2018).

This article is protected by copyright. All rights reserved 


\section{Aim of the Guideline}

This guideline aims to highlight the importance and need for scientific evidence in clinical decision making in the treatment of patients with periodontitis stages I to III. Its main objective is therefore, to support the evidence-based recommendations for the different interventions used at the different steps of periodontal therapy, based on the best available evidence and/or expert consensus. In so doing, this guideline aims to improve the overall quality of periodontal treatment in Europe, reduce tooth loss associated with periodontitis and ultimately improve overall systemic health and quality of life. A separate guideline covering the treatment of stage IV periodontitis will be published.

\section{Target users of the guideline}

Dental and medical professionals, together with all stakeholders related to health care, particularly oral health, including patients.

\section{Targeted environments}

Dental and medical academic / hospital environments, clinics and practices.

\section{Targeted patient population}

People with periodontitis stages I to III.

People with periodontitis stages I to III following successful treatment.

\section{Exceptions from the guideline}

This guideline did not consider the health economic cost-benefit ratio, since (i) it covers multiple different countries with disparate, not readily comparable health systems, and (ii) there is a paucity of sound scientific evidence available addressing this question. This guideline did not consider the treatment of gingivitis (although management of gingivitis is considered as an indirect goal in some interventions evaluated), the treatment of stage IV periodontitis, necrotising periodontitis, periodontitis as manifestation of systemic diseases and mucogingival conditions. 


\section{Methodology}

\section{General framework}

This guideline was developed following methodological guidance published by the Standing Guideline Commission of the Association of Scientific Medical Societies in Germany (AWMF) (https://www.awmf.org/leitlinien/awmf-regelwerk/awmf-guidance.html) and the Grading of Recommendations Assessment, Development and Evaluation (GRADE) Working Group (https://www.gradeworkinggroup.org/).

The guideline was developed under the auspices of the European Federation of Periodontology (EFP) and overseen by the EFP Workshop Committee. This guideline development process was steered by an Organizing Committee and a group of methodology consultants designated by the EFP. All members of the Organizing Committee were part of the EFP Workshop Committee.

To ensure adequate stakeholder involvement, the EFP established a guideline panel involving dental professionals representing 36 national periodontal societies within the EFP (Table 1a). These delegates were nominated, participated in the guideline development process and had voting rights in the consensus conference. For the guideline development process, delegates were assigned to four Working Groups that were chaired by the members of the Organizing Committee and advised by the methodology consultants. This panel was supported by key stakeholders from European scientific societies with a strong professional interest in periodontal care and from European organizations representing key groups within the dental profession, and key experts from non-EFP member countries, such as North America (Table 1b).

In addition, EFP engaged an independent guideline methodologist to advise the panel and facilitate the consensus process (Prof. Dr. med. Ina Kopp). The guideline methodologist had no voting rights.

EFP and the guideline panel tried to involve patient organizations but were not able to identify any regarding periodontal diseases at European Level. In a future update, efforts will be undertaken to include the perspective of citizens/patients (Brocklehurst et al., 2018). 


\section{Evidence Synthesis}

Systematic search and critical appraisal of guidelines

To assess and utilize existing guidelines during the development of the present guideline, wellestablished guideline registers and the websites of large periodontal societies were electronically searched for potentially applicable guideline texts:

- Guideline International Network (GIN)

- Guidelinecentral.com

- The National Institute for Health and Clinical Excellence (NICE)

- Canadian Health Technology Assessment (CADTH)

- European Federation for Periodontology (EFP)

- American Academy of Periodontology (AAP)

- American Dental Association (ADA)

The last search was performed on 30.09.2019. Search terms used were: "periodont*” Periodontal", “Guidelines, "Clinical Practice Guidelines". In addition, content was screened by hand searches. See Table 2.

Only guidelines published in English and with full texts available were included. The methodological quality of these guideline texts was critically appraised using the AGREE II framework (https://www.agreetrust.org/agree-ii/).

Most of the identified guidelines/documents were considered not applicable due to (i) their age,

(ii) their methodological approach, or (iii) their inclusion criteria. The recent German S3guideline was found to be potentially relevant, scored highest in the critical appraisal using AGREE II and was, therefore, used to inform the guideline development process.

\section{Systematic search and critical appraisal of the literature}

For this guideline, a total of 15 systematic reviews (SRs) were conducted to support the guideline development process (Carra et al., 2020; Dommisch, Walter, Dannewitz, \& Eickholz, 2020; Donos et al., 2019; Figuero, Roldan, et al., 2019; Herrera et al., 2020; Jepsen et al., 2019; Nibali et al., 2019; Polak et al., 2020; Ramseier et al., 2020; Salvi et al., 2019; Sanz-Sanchez et al., 2020; Slot, Valkenburg, \& van der Weijden, 2020; Suvan et al., 2019; Teughels et al., 2020; Trombelli et 
al., 2020). The corresponding manuscripts are published within this special issue of the Journal of Clinical Periodontology.

All SRs were conducted following the "Preferred Reporting Items for Systematic Reviews and Meta-Analyses" (PRISMA) framework (Moher, Liberati, Tetzlaff, \& Altman, 2009).

\section{Focused questions}

In all 15 systematic reviews, focused questions in $\mathrm{PICO}(\mathrm{S})$ format (Guyatt et al., 2011) were proposed by the authors in January 2019 to a panel comprising the working group chairs and the methodological consultants, in order to review and approve them (Table 3). The panel took great care to avoid overlaps or significant gaps between the SRs, so they would truly cover all possible interventions currently undertaken in periodontal therapy.

\section{Relevance of outcomes}

A narrative review paper was commissioned for this guideline (Loos \& Needleman, 2020) to evaluate the possible outcome measures utilized to evaluate the efficacy of periodontal therapy in relation to true patient-centred outcomes like tooth retention/loss. The authors found that the commonly reported outcome variable with the best demonstrated predictive potential for tooth loss was the reduction in periodontal probing pocket depth (PPD). Therefore, for this guideline, PPD reduction was used as primary outcome for those systematic reviews not addressing periodontal regeneration, and where tooth survival data were not reported. When reviewing regenerative interventions, gains in clinical attachment were used as the primary outcome measure. To avoid introducing bias by including possibly spurious findings of studies with very short follow-up, a minimal follow-up period of six months was requested for all reviews.

\section{Search strategy}

All SRs utilized a comprehensive search strategy of at least two different databases, supplemented by a hand search of periodontal journals and the reference lists of included studies.

In all SRs, the electronic and manual search, as well as the data extraction, was done in parallel by two different investigators. 


\section{Quality assessment of included studies}

In all SRs, the risk of bias of controlled clinical trials was assessed using the Cochrane Risk-of bias tool (https://methods.cochrane.org/bias/resources/rob-2-revised-cochrane-risk-bias-toolrandomized-trials). For observational studies, the Newcastle-Ottawa-Scale was used http://www.ohri.ca/programs/clinical_epidemiology/oxford.asp.

\section{Data synthesis}

Where applicable, the available evidence was summarized by means of meta-analysis, or other tools aimed for pooling data (network meta-analysis, Bayesian network meta-analysis).

\section{From Evidence to Recommendation: Structured Consensus Process}

The structured consensus development conference was held during the XVI European Workshop in Periodontology in La Granja de San Ildefonso Segovia, Spain, on November 10th - 13th, 2019. Using the 15 SRs as background information, evidence-based recommendations were formally debated by the guideline panel using the format of a structured consensus development conference, consisting of small group discussions and open plenary were the proposed recommendations were presented, voted and adopted by consensus and (Murphy et al., 1998).

In the small group phase, delegates convened in four working groups addressing the following subtopics; 1) “periodontitis stages I \& II”; 2) “periodontitis stage III”; 3) “periodontitis stage III with intraosseous defects and/or furcations", and 4) "supportive periodontal care". This working groups were directed by two chairpersons belonging to the EFP Workshop Committee. With the support of an expert in methodology in each working group, recommendations and draft background texts were generated and subsequently presented, debated and put to a vote in the plenary of all delegates. During these plenary sessions. the guideline development process and discussions and votes were overseen and facilitated by the independent guideline methodologist (I.K.). The plenary votes were recorded using an electronic voting system, checked for plausibility in then introduced into the guideline text.

The consensus process was conducted as follows:

\section{Plenary 1}


Introduction to guideline methodology (presentation, discussion) by the independent guideline methodologist (I.K.).

\section{Working group Phase 1}

- Peer evaluation of declarations of interest and management of conflicts.

- Presentation of the evidence (SR results) by group chairs and methodology consultants.

- Invitation of all members of the working group to reflect critically on the quality of available evidence by group chairs, considering GRADE criteria.

- Structured group discussion:

○ development of draft recommendation and their grading, considering GRADEcriteria.

○ development of draft background texts, considering GRADE-criteria.

○ invitation to comment draft recommendations and background text to suggest reasonable amendments by group chairs.

$\circ$ collection and merging of amendments by group chairs.

○ initial voting within the working group on recommendations and guideline text to be presented as group result in the plenary.

\section{Plenary 2}

- Presentation of working group results (draft recommendations and background text) by Working Group chairs.

- Invitation to formulate questions, statements and reasonable amendments of the plenary by the independent guideline methodologist /facilitator.

- Answering of questions by working group chairpersons.

- Collection and merging of amendments by independent moderator.

- Preliminary vote on all suggestions provided by the working groups and all reasonable amendments.

- Assessment of the strength of consensus.

- Opening debate, where no consensus was reached or reasonable need for discussion was identified.

- Formulation of tasks to be solved within the working groups.

\section{Working Group Phase 2}


- Discussion of tasks and potential amendments raised by the plenary.

- Formulation of reasonable and justifiable amendments, considering the GRADE framework.

- Initial voting within the working group on recommendations and guideline text for plenary.

\section{Plenary 3}

- Presentation of working group results by working group chairpersons.

- Invitation to formulate questions, statements and reasonable amendments of the plenary by the independent moderator.

- Collection and merging of amendments by independent moderator.

- Preliminary vote.

- Assessment of the strength of consensus.

- Opening debate, where no consensus was reached or reasonable need for discussion was identified.

- Formulation of reasonable alternatives.

- Final vote of each recommendation.

\section{Definitions: Rating the Quality of Evidence, Grading the Strength of Recommendations and Determining the Strength of Consensus}

For all recommendations and statements, this guideline makes transparent

- the underlying quality of evidence, reflecting the degree of certainty / uncertainty of the evidence and robustness of study results

- the grade of the recommendation, reflecting criteria of considered judgement the strength of consensus, indicating the degree of agreement within the guideline panel and thus, reflecting the need of implementation

\section{Quality of Evidence}

The quality of evidence was assessed using a recommended rating scheme (Balshem et al., 2011;

Schunemann, Zhang, Oxman, \& Expert Evidence in Guidelines, 2019).

\section{Strength of Recommendations}


The grading of the recommendations used the grading scheme (Table 4) by the (German Association of the Scientific Medical Societies (AWMF) \& Standing Guidelines Commission, 2012), taking into account not only the quality of evidence, but also considered judgement, guided by and the following criteria:

- relevance of outcomes and quality of evidence for each relevant outcome

- consistency of study results

- directness regarding applicability of the evidence to the target population/PICO specifics

- precision of effect estimates regarding confidence intervals

- magnitude of the effects

- balance of benefit and harm

- ethical, legal, economic considerations

- patient preferences

The grading of the quality of evidence and the strength of a recommendation may therefore differ in justified cases.

\section{Strength of Consensus}

The consensus determination process followed the recommendations by the (German Association of the Scientific Medical Societies (AWMF) \& Standing Guidelines Commission, 2012). In case, consensus could not be reached, different points of view were documented in the guideline text. See Table 5.

\section{Editorial Independence}

\section{Funding of the guideline}

The development of this guideline and its subsequent publication was financed entirely by internal funds of the European Federation of Periodontology, without any support from industry or other organisations.

\section{Declaration of Interests and Management of Potential Conflicts}

All members of the guideline panel declared secondary interests using the standardized form provided by the International Committee of Medical Journal Editors (ICMJE) (International Committee of Medical Editors). 
Management of conflicts of interests (CoI) was discussed in the working groups, following the principles provided by the Guidelines International Network (Schunemann et al., 2015).

According to these principles, panel members with relevant, potential CoI abstained from voting on guideline statements and recommendations within the consensus process.

\section{Peer review}

All 15 systematic reviews, and the position paper on outcome variables commissioned for this guideline, underwent a multi-step peer review process. First, the draft documents were evaluated by members of the EFP Workshop Committee and the methodological consultants using a custommade appraisal tool to assess (i) the methodological quality of the SRs using the AMSTAR 2 checklist (Shea et al., 2017), and (ii) whether all PICO(S) questions were addressed as planned. Detailed feedback was then provided for the SR authors. Subsequently, all 15 systematic reviews and the position paper underwent the regular editorial peer review process defined by the Journal of Clinical Periodontology.

The guideline text was drafted by the chairs of the working groups, in close cooperation with the methodological consultants, and circulated in the guideline group before the workshop. The methodological quality was formally assessed by an outside consultant using the AGREE framework. The guideline was subsequently peer reviewed for its publication in the Journal of Clinical Periodontology following the standard evaluation process of this scientific journal

\section{Implementation and dissemination plan}

For this guideline, a multi-stage dissemination and implementation strategy will be actioned by the EFP, supported by a communication campaign.

\section{This will include:}

- Publication of the guideline and the underlying systematic reviews and position paper as an Open Access special issue of the Journal of Clinical Periodontology

- Local uptake from national societies, either by Commentary, Adoption, or Adaptation (Schunemann et al., 2017) 
- Generation of educational material for dental professionals and patients, dissemination via the EFP member societies

- Dissemination via educational programs on dental conferences

- Dissemination via EFP through European stakeholders via National Societies, members of EFP

- Long-term evaluation of the successful implementation of the guideline by poll of EFP members

The timeline of the guideline development process is detailed in Table 6.

\section{Validity and update process}

The guideline is valid until 2025. However, the EFP, represented by the members of the Organizing Committee, will continuously assess current developments in the field. In case of major changes of circumstances, e.g. new relevant evidence, they will trigger an update of the guideline to potentially amend the recommendations. It is planned to update the current guideline regularly on demand in form of a living guideline. 